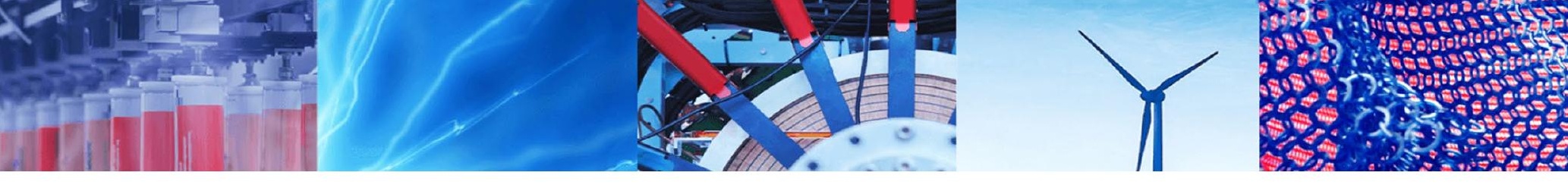

Research Article

\title{
Investigating the growth of AIGaN/AIN heterostructure by modulating the substrate temperature of AIN buffer layer
}

\author{
Neha Aggarwal ${ }^{1}$. Shibin Krishna ${ }^{2}$. Lalit Goswami ${ }^{1}$. Shubhendra Kumar Jain ${ }^{1,3,6}$ • Akhilesh Pandey ${ }^{4}$. \\ Abhiram Gundimeda ${ }^{5} \cdot$ Pargam Vashishtha ${ }^{1,6}$. Jasveer Singh ${ }^{1} \cdot$ Sandeep Singh ${ }^{1}$. Govind Gupta ${ }^{1,6}$
}

Received: 12 November 2020 / Accepted: 25 January 2021 / Published online: 8 February 2021

(C) The Author(s) 2021 OPEN

\begin{abstract}
We have investigated the impact of AIN buffer layer growth parameters for developing highly single crystalline AIGaN films. The low mobility of Al adatoms and high temperature for compound formation are amongst the major causes that affects the growth quality of AIGaN films. Thus, proper optimization need to be carried out for achieving high quality AlGaN due to an augmented tendency of defect generation compared to GaN films. Thus, growth conditions need to be amended to maximize the incorporation ability of adatoms and minimize defect density. So, this study elaborates the growth optimization of AIGaN/AIN/Si (111) heterostructure with varied AIN buffer growth temperature $\left(760\right.$ to $\left.800^{\circ} \mathrm{C}\right)$. It was observed that the remnant Al in low temperature growth of AIN buffer layer resist the growth quality of AIGaN epitaxial films. A highly single crystalline AIGaN film with comparatively lowest rocking curve FWHM value $\left(\sim 0.61^{\circ}\right)$ and smooth surface morphology with least surface defect states was witnessed when AIN buffer was grown at $780^{\circ} \mathrm{C}$. From the Vegard's law, the photoluminescence analysis unveils Aluminium composition of $31.5 \%$ with significantly reduced defect band/NBE band ratio to 0.3 . The study demonstrates good crystalline quality AIGaN film growth with Aluminium content variation between $\sim 30-39 \%$ in AIGaN/AIN heterostructure on Si(111) substrate leading to a bandgap range which is suitable for next-generation solar-blind photodetection applications.
\end{abstract}

Keywords AIGaN film · Epitaxial growth · PAMBE · Crystalline quality · Al incorporation

\section{Introduction}

A ternary alloy of III-Nitride material system i.e. $\mathrm{Al}_{\mathrm{x}} \mathrm{Ga}_{1-\mathrm{x}} \mathrm{N}$ has huge number of potential applications in high power and radiation-resistance electronics such as flame and heat detectors, missile plume detection and safe inter-satellite communications, UV calibration and monitoring devices for medical and biological sensors, etc. [1-3]. In the past few years, these applications were fulfilled by photomultiplier tubes, SiC and Si based detectors [4]. Sooner the photomultiplier tubes are found to be inefficient due to bulky instrumentation while, Si, having a narrow bandgap of $1.1 \mathrm{eV}$, demands optical filters to function as a UV detector which again makes the system massive and costly. However, bandgap of $\mathrm{Al}_{\mathrm{x}} \mathrm{Ga}_{1-\mathrm{x}} \mathrm{N}$ can be tuned between $3.4 \mathrm{eV}$ (at $0 \% \mathrm{Al}$ ) and $6.2 \mathrm{eV}$ (at $100 \% \mathrm{Al}$ ) thus; it offers a great advantage over Si by

Supplementary information The online version of this article (https://doi.org/10.1007/s42452-021-04274-4) contains supplementary material, which is available to authorized users.

$\checkmark$ Govind Gupta, govindnpl@gmail.com | 'CSIR-National Physical Laboratory (NPL), Dr. K. S. Krishnan Marg, New Delhi 110012, India. ${ }^{2}$ Advanced Semiconductor Laboratory, King Abdullah University of Science and Technology, Thuwal 23955 , Saudi Arabia. ${ }^{3}$ Functional Materials and Microsystems Research Group and the Micro Nano Research Facility, RMIT University, Melbourne, VIC 3000, Australia. ${ }^{4}$ Solid State Physics Laboratory, Defence Research and Development Organization, Timarpur, Delhi 110054, India. ${ }^{5}$ Department of Materials Science and Metallurgy, University of Cambridge, Cambridge CB3 OFS, UK. ${ }^{6}$ Academy of Scientific and Innovative Research, CSIR-HRDC Campus, Ghaziabad, Uttar Pradesh 201002, India. 
eliminating the need of optical filters [5]. Besides the robustness of $\mathrm{Al}_{\mathrm{x}} \mathrm{Ga}_{1-\mathrm{x}} \mathrm{N}$ material, it also provides the potential to yield stable device operation even in harsh environments $[6,7]$. On the other hand, SiC, having a bandgap of $\sim 3.0 \mathrm{eV}$ is similar in robustness to nitrides, still does not have the potential to tune the bandgap via alloying and thus, they require expensive filters for UV applications $[5,8]$. Since, an ideal optoelectronic device necessitates deeper understanding about various factors affecting optical and electrical transport properties of the thin films. The major challenge that hinders the development of highly efficient AIGaN/AIN heterostructure based devices is the high quality growth on suitable substrate as this will directly impact the structural and optical properties which ultimately alter the current transport behavior in the device. Usually, high quality AIGaN has been grown on sapphire substrates or GaN templates for better growth quality [9-11] but soon researchers started growing AlGaN on Si substrates for better integration with existing established device fabrication technology [12-15]. Here, Si substrate is utilized because it is easily available at low cost \& provides integration with the well-established Si growth technology that ensures high quality device fabrication as well. However, the direct growth of AlGaN on $\mathrm{Si}$ substrate may lead to high amount of defects and cracks due to lattice-mismatch and thermal expansion coefficient mismatch between the substrate and the epitaxial film [5]. Consequently, these defects states instigate degradations in structural, morphological and optical properties. Thus, introduction of a buffer layer is extremely necessary as it plays a key role in improving the crystalline quality and reducing the defect states in epitaxial AIGaN films grown by plasmaassisted molecular beam epitaxy (PAMBE) system $[16,17]$. However, most of the studies report AIGaN growth on $\mathrm{Si}$ using $\mathrm{GaN}$ buffer layer and only few reports elaborate the growth on Si using AIN buffer $[3,18]$. Although, the effect of AIN buffer layer has been vastly studied $[19,20]$ on the properties of GaN films still its role on AIGaN/AIN heterostructure grown on $\mathrm{Si}$ (111) has not yet explored much in detail. Hence, this work explicates the influence of AIN buffer layer on the crystalline quality and defect density of epitaxially grown AIGaN films by PAMBE. The present study also correlates the role of different AIN buffer growth temperatures with the structural, morphological and optical properties of AlGaN films including compositional variations that result in bandgap variation as well.

\section{Materials and methods}

The AIGaN/AIN heterostructures were grown on $\mathrm{p}-\mathrm{Si}$ (111) substrate $(325 \pm 25 \mu \mathrm{m}$ thick, boron doped (p-type) with resistivity of $5-15 \Omega-\mathrm{cm}$ ) by PAMBE system. The (111) orientation of Si substrate is utilized in this study because the growth of wurtzite hexagonal AIN (002) is more preferred on Si (111) over other orientations of silicon substrate which leads to good quality nitride heterostructure [21]. The silicon substrates were chemically pre-cleaned by using the standard RCA procedure before loading into the load-lock chamber followed by out-gassing in the buffer chamber at $630^{\circ} \mathrm{C}$.

Prior to growth, the Si substrates were flashed at high temperature in the growth chamber to achieve atomically clean $7 \times 7$ reconstructed surfaces. Initially, $480 \mathrm{~nm}$ thick AIN buffer layer is grown on Si substrate since, the introduction of AIN buffer layer may lead towards crack free growth. Thus, a number of samples have been grown with varied growth parameters of AIN and some of them are discussed in this study to avail better understanding about role of buffer layer growth conditions on the AIGaN epitaxial layer. A growth-time vs temperature diagram representing each step involved in the growth process is shown in Fig. 1. The diagram illustrates that the growth procedure starting from the outgassing of substrate to obtaining an epitaxial AlGaN heterostructure takes nearly $330 \mathrm{~min}$. to complete. Here, three samples are being discussed which are grown with different AIN substrate temperatures varied between 760 to $800^{\circ} \mathrm{C}$ and labelled according to buffer growth temperature i.e., S-760, S-780 and S-800. Apart from the substrate temperature of AIN, rest all other growth parameters are kept constant in complete growth process of all the samples. Then, $\sim 1.5 \mu \mathrm{m}$ thick AIGaN has been grown with a constant pre-optimized parameters on the AIN buffer with varied growth temperature. The substrate temperature, BEP of $\mathrm{Ga}$ and $\mathrm{Al}, \mathrm{N}_{2}$ flux as well as RF power and growth time during $\mathrm{AlGaN}$ growth was set to $690^{\circ} \mathrm{C}, 2 \times 10^{-6}$ Torr, $2 \times 10^{-7}$ Torr, $3 \mathrm{sccm}, 400 \mathrm{~W}$ and $120 \mathrm{~min}$, respectively. Therefore, the effects of AIN buffer layer were elaborated via various structural, morphological and optical properties of PAMBE grown epitaxial AIGaN film.

The structural quality of the grown films was gauged by using high resolution X-ray diffraction (HRXRD, Panalytical X'Pert PRO MRD System), and the surface morphology was probed by atomic force microscopy (AFM, Multimode-V Veeco) and FESEM (ZEISS AURIGA). The optical properties, bandgap evaluations and stress variations have been reckoned by using photoluminescence (PL) and Raman spectroscopy (RS), respectively. PL measurements were carried out using FLS980 D2D2 (Edinburg) system equipped with a $\mathrm{He}-\mathrm{Cd}$ laser operating at $325 \mathrm{~nm}$ as an excitation source. Room temperature (RT) micro-Raman measurements were performed using a triple Raman spectrometer in backscattering configuration with an excitation source of argon ion laser $(\mathrm{Ar}+)$ having an operating wavelength of $514 \mathrm{~nm}$. 
Fig. 1 The growth-time-temperature diagram representing complete timeline of developing epitaxial AlGaN on Si (111) substrate

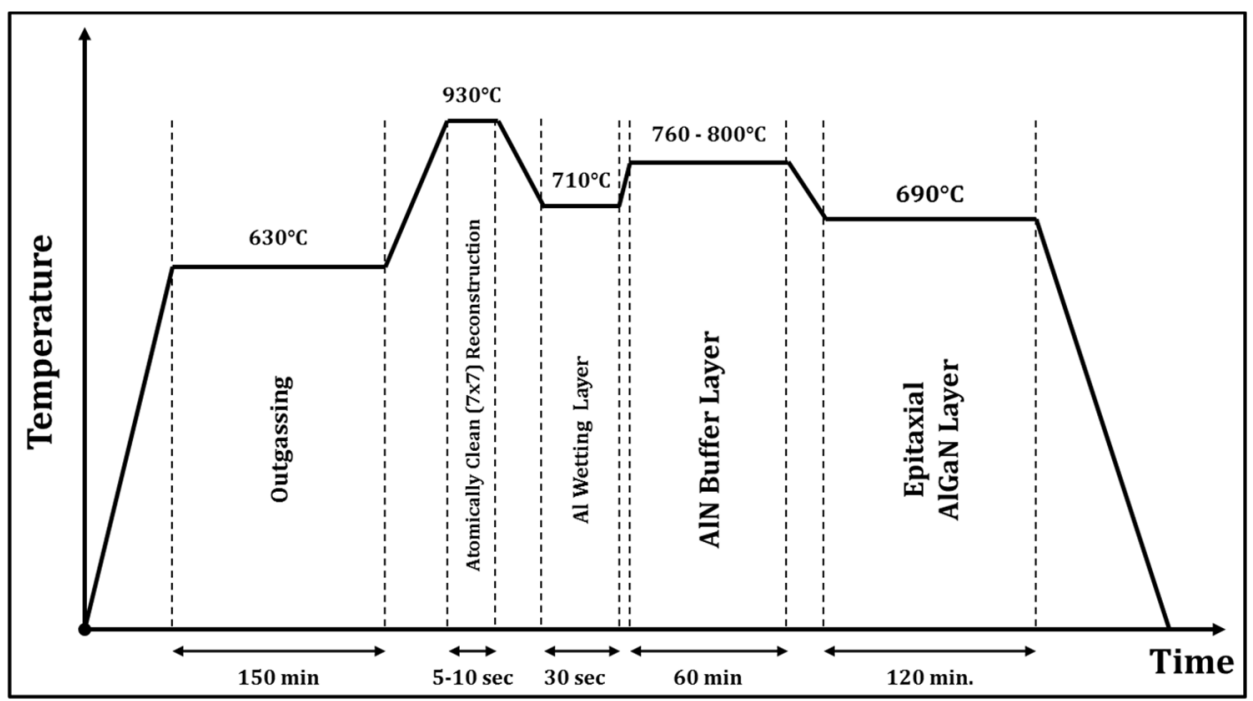

\section{Results and discussions}

A systematic study of the hetero-epitaxial growth of AIGaN/AIN on Si (111) via PAMBE has been illustrated by using a number of samples grown with varied buffer layer growth parameters. Depending upon the buffer layer growth conditions, physical as well as optical properties of the epitaxial AIGaN film may differ. Several properties of the grown heterostructure is analyzed deeply and discussed as follows.

\subsection{Structural properties}

A schematic diagram showed in Fig. 2a represents the PAMBE grown heterostructure where initially AIN film is grown on atomically cleaned $\mathrm{Si}(111)$ surface which act as buffer layer for the epitaxial growth of AIGaN film. The AIGaN films grown on the three distinct AIN buffer layers has been characterized by HRXRD measurements to examine the quality of grown AIGaN/AIN/Si (111) heterostructure. Figure $2 b$ illustrates the HRXRD $2 \theta$-omega scan of S-760 sample where the highly intense and sharp peaks at $35.04^{\circ}$ and $36.08^{\circ}$ explicates the growth of single crystalline AIGaN and AIN films, respectively. Moreover, the presence of first and second order X-ray diffractions of AIGaN in the $2 \theta$-omega scan endorses that single crystalline AIGaN film has been successfully grown epitaxially on the Si substrate. Besides, the sharp peaks at $28.4^{\circ}$ and $58.8^{\circ}$ have been derived from the first and second order diffractions of Si (111). An additional peak at $\sim 31.8^{\circ}$ has been observed in Fig. $2 \mathrm{~b}$ and Fig. $2 \mathrm{c}$ which is found to be associated with the (002) orientation of $\mathrm{a}-\mathrm{Si}_{3} \mathrm{~N}_{4}$ that might have formed while exposing the substrate to active $\mathrm{N}$ species for AIN growth [22].
Further, HRXRD analysis of S-780 sample (Fig. 2c) indicates that high temperature buffer can yield improved crystalline quality of AIGaN by observing very sharp peaks with reduced FWHM value. It has been clearly witnessed that the intensity ratio of AIGaN/AIN in S-760 (9.75) is more compared to S-780 sample (3.48). This can be attributed to a slight remnant Al present in low temperature $\left(760^{\circ} \mathrm{C}\right)$ grown AIN as confirmed from a small peak at $38.1^{\circ}$ in $2 \theta$-omega scan of S-760 (in Fig. 2 (b) which was absent in the sample $\mathrm{S}-780$ where AIN is grown at a slightly higher temperature $\left(780^{\circ} \mathrm{C}\right)$ leading to improved AIN quality as well as AIGaN epitaxial quality. This signifies that the remnant Al (in S-760) must have resisted the growth of AIGaN film thereby, affecting its growth rate and quality. However, upon further increasing the AIN buffer growth temperature to $800^{\circ} \mathrm{C}$ in S-800 sample, HRXRD analysis shown in Fig. 2d) reveals that such high temperature buffer can lead to a reduction in peak intensity of AIGaN as well as AIN as observed from the plot peaks in S-800 with reduced intensity compared to S-780. This analysis reveals that higher growth temperature results in lower growth rate of AIN buffer which slows down the growth rate of AIGaN epitaxial film as well. Thus, the sharp and intense peaks signify highly single crystalline nature of AIN and AIGaN films in each grown heterostructure optimized to a best crystallinity and highest intensity in S-780 sample.

For better understanding, the average grain size for AIN and AIGaN layers has been evaluated by using the Scherrer formula: $[20,23]$

$\mathrm{t}=\mathrm{K} \cdot \lambda / \beta \cos \theta$

Here, ' $\mathrm{t}$ ' is the average grain size, ' $\mathrm{K}$ ' is a proportionality constant (assumed value near to unity), ' $\lambda$ ' is the wavelength of the incident beam $(0.15406 \mathrm{~nm}),{ }^{\prime} \beta^{\prime}$ is the 
Fig. 2 a A schematic diagram of heterostructure grown via PAMBE; HRXRD 20-omega scans of grown epitaxial structure in b S-760;c S-780 and d S-800

Table 1 The evaluated average grain sizes of AIN and AIGaN grown with varied buffer layer temperatures

\begin{tabular}{llll}
\hline Material & \multicolumn{2}{l}{$\begin{array}{l}\text { Average grain size } \\
(\mathrm{nm})\end{array}$} \\
\cline { 2 - 4 } & S-760 & S-780 & S-800 \\
\hline AIN & 24.75 & 49.97 & 44.82 \\
AIGaN & 26.72 & 52.05 & 49.02 \\
\hline
\end{tabular}

(a)
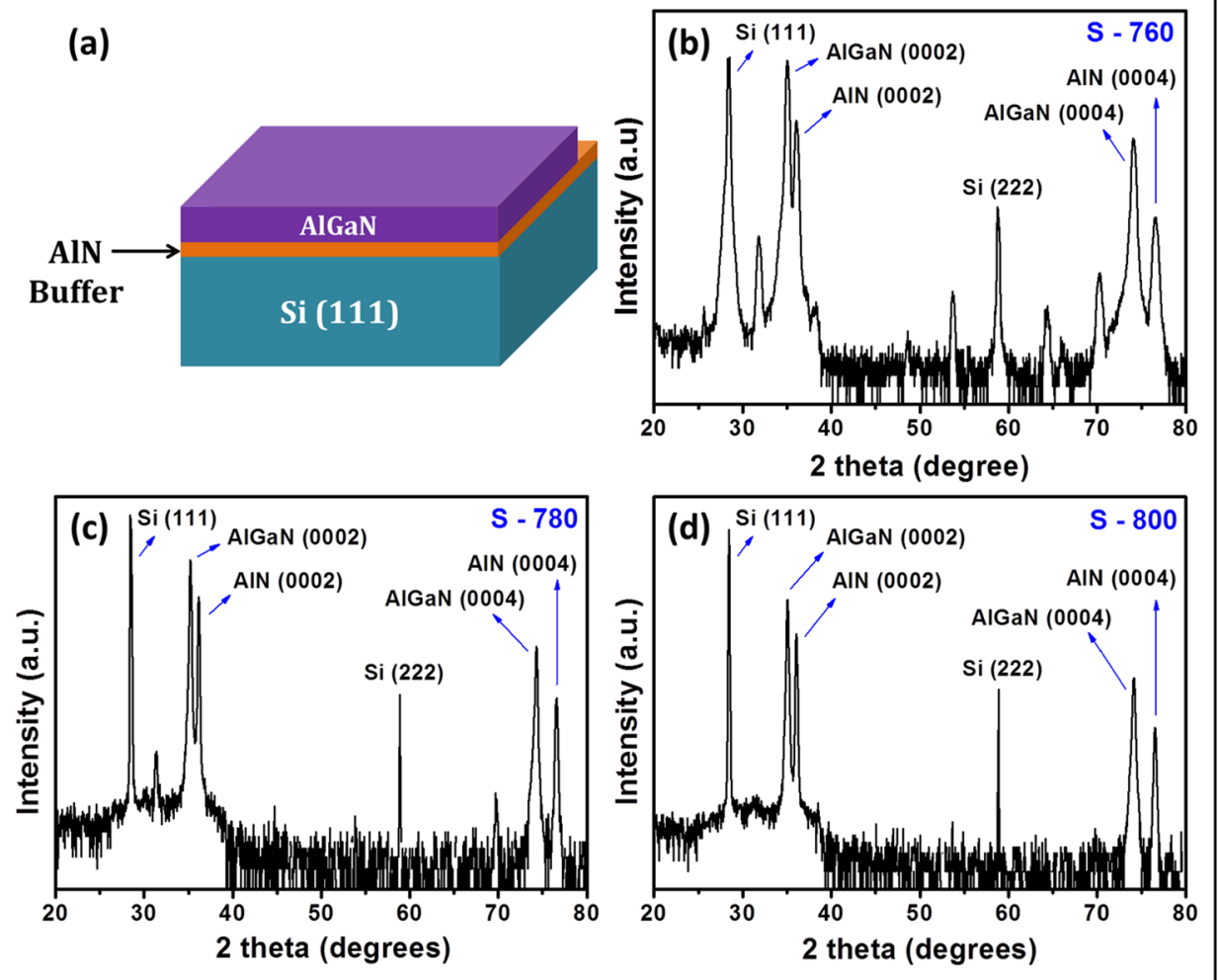

FWHM of the AIN and AIGaN peak from $2 \theta$-omega scan of HRXRD pattern along (0002) plane of diffraction (in radians), and ' $\theta$ ' is the diffraction angle of the AIN (0002) and AIGaN (002) peaks. The grain sizes obtained by the Scherrer formula are tabulated in Table 1.

It was clearly observed from Table 1 that the average grain size is largest for the AIN and AIGaN grown in $\mathrm{S}-780$ sample which is suggesting that the buffer growth temperature of $780^{\circ} \mathrm{C}$ is providing adequate amount of surface energy to the ad-atoms arriving at the surface for growing the desired nitride heterostructure. Next, to quantify the crystalline quality of grown films, the $\mathrm{X}$-ray rocking curves (XRCs) has been utilized which are plotted in Fig. $3 \mathrm{a}$ where the evaluated FWHM value is $0.63^{\circ}, 0.61^{\circ}$ arcsec and $0.93^{\circ}$ along in-plane (002) diffraction from samples S-760, S-780 and S-800, respectively (Fig. 3b). The FWHM values are directly related to the dislocation density existing in the grown layer which will lately govern the performance of an optoelectronic

Fig. 3 a The comparative XRC plots of AlGaN along (0002) plane of diffraction; $\mathbf{b}$ The trends revealing variation in FWHM from XRCs and strain evaluated for epitaxial AIGaN films with changing buffer growth temperatures

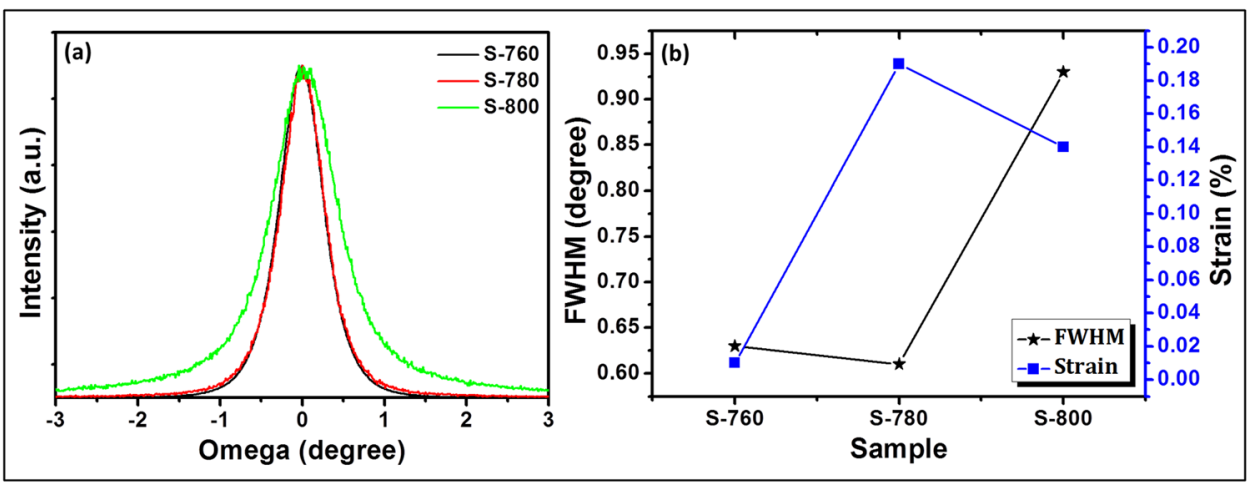


device to be developed using them [24]. It is interesting to notice that the FWHM value of S-780 sample is lower than other two samples grown with varied buffer via PAMBE and some previous reports of AIGaN growth on $\mathrm{Si}$ (111) [25]. The lower value of the FWHM in sample S-780 exemplifies low mosaicity of the grown film and can be correlated to better formation of AIN which resulted in enhanced crystalline quality of the epitaxial AIGaN film. The FWHM and strain variation is represented in Fig. $3 \mathrm{~b}$ where the strain values have been evaluated by using the peak positions of 2 $\theta$-omega scans in Bragg's law [26].

Here, the strain free values were assumed to be the lattice constants corresponding to the Al compositions evaluated from omega- $2 \theta$ scans which will be discussed next in this section. Upon quantifying, the strain values are found to be $0.01 \mathrm{GPa}, 0.19 \mathrm{GPa}$ and $0.14 \mathrm{GPa}$ for S-760, S-780 and S-800, respectively. The higher strain value in S-780 implies strained film which may or may not lead to cracks that can further be affirmed by observing the morphological properties of the grown AIGaN films. The topographical study has been discussed in next section for gaining deeper understanding into it. Before that, the omega-2 theta scan has been carried out for the determination of $\mathrm{Al}$ composition in PAMBE grown AIGaN films. Figure $4 a-c$ shows the omega- 2 theta scan HRXRD spectra along the symmetric (0002) plane of diffraction from the AIGaN and AIN films.
The fitting of these spectra (not shown here) revealed the Al composition of $37.5 \%, 39 \%$ and $33 \%$ in sample S-760, S-780 and S-800, respectively. The evaluated Al composition variation is plotted against sample in Fig. $4 \mathrm{~d}$. It was noticed that the Al composition is highest in sample S-780 due to optimized buffer conditions which lead to better AIN quality and thereby, enhanced AIGaN crystallinity along with higher Al incorporation. The physical mechanism and surface kinetics defined by the buffer growth temperature towards increasing the Al incorporation in S-780 sample is explained as follows. At lower growth temperature $\left(760^{\circ} \mathrm{C}\right)$ of AIN buffer layer, the HRXRD analysis of $2 \theta$-omega scan shows a small peak at $\sim 38.20^{\circ}$ which is associated with the presence of excess metallic Al at the interface. [3] This is due to inadequate surface energy at lower temperature which limits the conversion of all the arriving $\mathrm{Al}$ ad-atoms into AIN compound, resulting in remnant $\mathrm{Al}$ at the interface. So, when the surface is exposed to $\mathrm{Al}, \mathrm{Ga}$ ad-atoms and $\mathrm{N}$ active species for AIGaN growth, it was observed that initially the excess Al utilizes the nitrogen to form AIN restricting the formation of AIGaN and later on, the growth of AIGaN was pursued leading to lower Al incorporation in the $\mathrm{AlGaN}$ layer. Secondly, at the higher temperature of $800^{\circ} \mathrm{C}$, nitrogen rich condition leads to III-group vacancies. Upon exposing the surface with $\mathrm{Al}, \mathrm{Ga}$ and $\mathrm{N}$ for AlGaN growth, these vacancies are initially filled by III-group elements which should be
Fig. 4 HRXRD omega-2theta scans of epitaxial AlGaN films grown in a S-760; b S-780 and c S-800 samples; $\mathbf{d}$ Al compositions evaluated by fitting of omega- $2 \theta$ scans
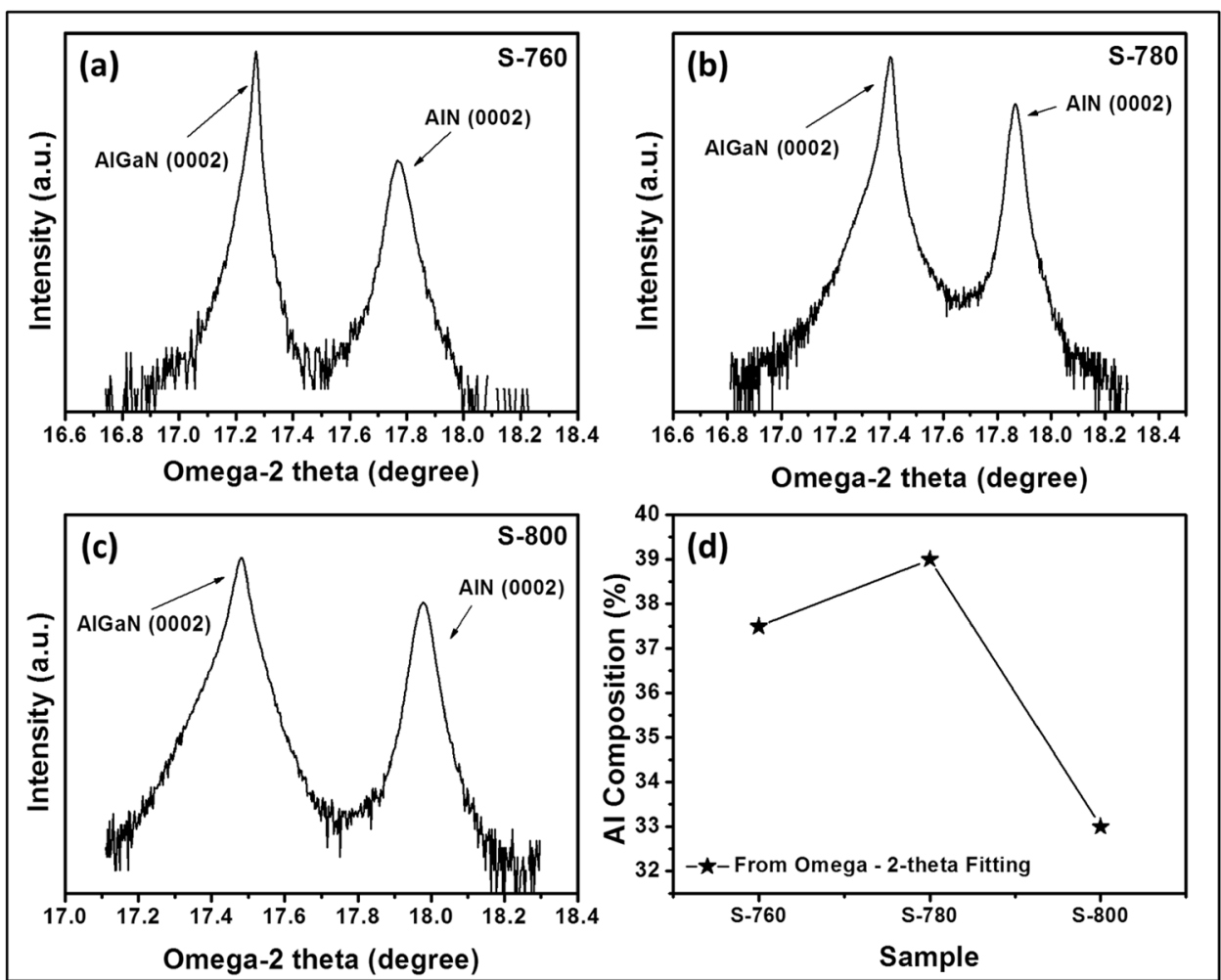
followed by the AIGaN growth. This leads to lower incorporation of $\mathrm{Al}$ in $\mathrm{AlGaN}$ layer since the growth time and Al flux is kept constant in all the three samples. Finally, in the stoichiometric conditions of sample S-780, there was no remnant $\mathrm{Al}$ after AIN deposition and also no III-group vacancies due to optimum growth temperature of AIN buffer layer. This leads to smooth and quick start for the growth of AIGaN as soon as the surface was exposed to the $\mathrm{Al}, \mathrm{Ga}$ and $\mathrm{N}$ adatoms. This must resulted in highest incorporation of $\mathrm{Al}$ in the $\mathrm{AIGaN}$ grown in $S-780$. Next, the influence of buffer quality on surface morphology of AIGaN has been examined by analyzing the FESEM and AFM micrographs shown in Fig. 5.

\subsection{Morphological properties}

Figure 5 displays the topographical features of the top AIGaN surface in all the grown AIGaN/AIN heterostructures. The influence of buffer growth temperature has been clearly witnessed by the variations in surface morphology of AIGaN. The FESEM, AFM and depth profiles are shown in Fig. $5(\mathrm{a}, \mathrm{d}, \mathrm{g}),(\mathrm{b}, \mathrm{e}, \mathrm{h})$ and $(\mathrm{c}, \mathrm{f}, \mathrm{i})$ for the AIGaN surface in samples S-760, S-780 and S-800, respectively. It can be observed from the FESEM image of S-760 (Fig. 5a) that the surface is quite smooth with the presence of few nano-islands while, a highly smooth surface with almost coalesced epitaxial film is witnessed in the FESEM image of S-780 (Fig. $5 \mathrm{~d}$ ) and the nano-islands observed in S-760 has also been reduced effectively. However, when the AIN growth temperature increased to $800{ }^{\circ} \mathrm{C}$ in S-800,
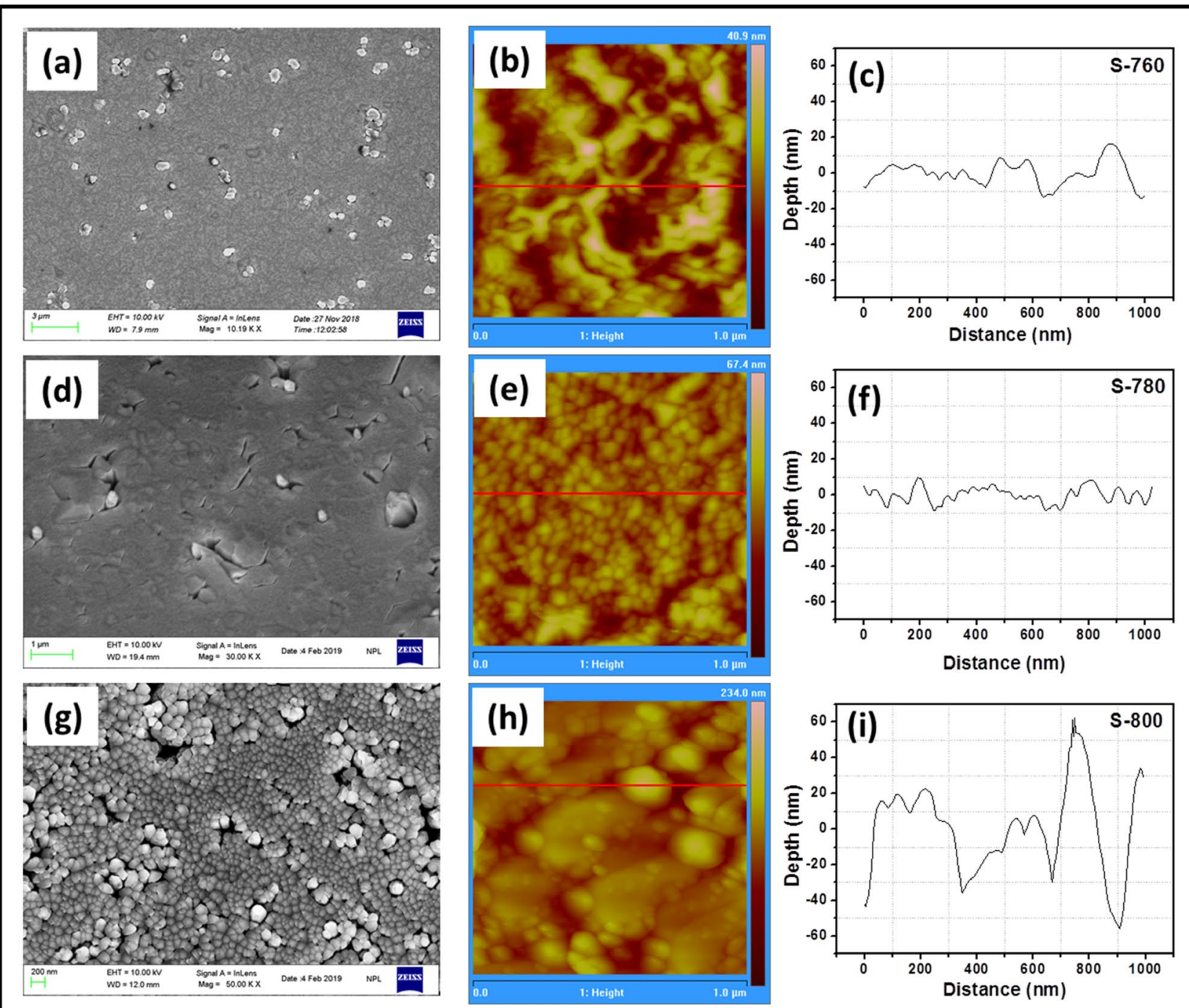

Fig. 5 The topographical analysis using (a,d,g) FESEM; (b,e,h) AFM and (c,f,i) Depth profiling (via AFM where the area is marked by red line) for samples S-760, S-780 and S-800, respectively

\section{SN Applied Sciences}


the surface becomes quite rough with huge nano-sized islands agglomerated into an epitaxial film as shown in Fig. 5g. Therefore, it can be concluded that the film grown in sample S-780 has smoother surface morphology along with less surface defects than the films grown in samples S-760 and S-800.

Furthermore, to avail better understanding of morphological properties, the surface was probed by AFM which will divulge the surface morphology along with surface roughness and its depth profile. The AFM image of AIGaN film grown in S-760 (Fig. 5b) shows slightly smooth surface morphology (r.m.s. roughness, $R_{\text {r.m.s }}=6.44 \mathrm{~nm}$ ) covered with small troughs which were assumed to be appeared due to the nitrogen vacancies in epitaxial AIN buffer layer that was grown in Al-rich conditions [27]. As the buffer growth temperature increases to $780^{\circ} \mathrm{C}$ (Fig. 5e), the active nitrogen species is expected to be entirely consumed by the impinging Al flux due to sufficiently available thermal energy on the substrate and thereby, growing high quality AIN layer effectively improved the surface morphology of AIGaN layer to a greater extent [28]. An effective reduction in surface roughness has been witnessed with the $R_{r . m . s}$ value of $5.62 \mathrm{~nm}$ which may lead to better carrier transport if utilized for device fabrication. This has been correlated with the high strain value evaluated from HRXRD that mutually explicate highly smooth and slightly strained film surface by restricting the crack formation which sometimes appear due to strain relaxation. Recently, Singh et al. reported that a reduction in roughness of AIN buffer layer could lead to improved mobility resulting in enhanced transport properties [29]. However, further increment in buffer growth temperature to $800^{\circ} \mathrm{C}$ resulted in morphological transition in AIGaN film from highly smooth surface to a comparatively rough surface as shown in Fig. 5h. The surface is pertaining large amount of nano-sized islands agglomerated and the surface roughness is found to be increased nearly 3 times having $R_{\text {r.m.s }}$ value of $18.2 \mathrm{~nm}$. The surface degradation could be associated to a limited incorporation probability of $\mathrm{Al}$ in AIN due to higher substrate temperature and thus lead toward a comparatively degraded quality of AIN buffer layer. This belong to a rise in excess active $\mathrm{N}$ on the surface of the film due to increased surface diffusion barrier, that resulted in N-rich growth of buffer layer leading to degraded morphology of AIGaN epitaxial layer grown on it. Further, the depth profile observed for each AIGaN film revealed smoothest surface in AIGaN grown in S-780 (Fig. 5f) among all the samples grown via PAMBE under different buffer growth conditions.

\subsection{Optical properties}

The influence of buffer layer parameters on optical properties has been explored which will indirectly play a prominent role in deciding the behavior of optoelectronic devices to be developed using $\mathrm{AIGaN}[3,30]$. Figure 6a represents the RT -PL spectra of each sample where a sharp NBE emission is observed for every AIGaN film with a trivial shift in its NBE position leading to slight variation in the
Fig. 6 a The RT-PL spectra acquired from the AlGaN films grown in S-760, S-780 and $\mathrm{S}-800$ samples; $\mathbf{b}$ the plot showing variation in $\mathrm{Al} \mathrm{com-}$ position with changing buffer condition in each sample; $c$ the trends of PL NBE bandwidth and ratio of DB to NBE intensity variation $\left(\mathrm{I}_{\mathrm{DB}} / \mathrm{I}_{\mathrm{NBE}}\right)$ against each sample grown with different buffer parameters; $\mathbf{d}$ The micro-Raman Spectra enlarged for the range of $550-750 \mathrm{~cm}^{-1}$; inset shows the complete Raman spectra in the range of $450-800 \mathrm{~cm}^{-1}$

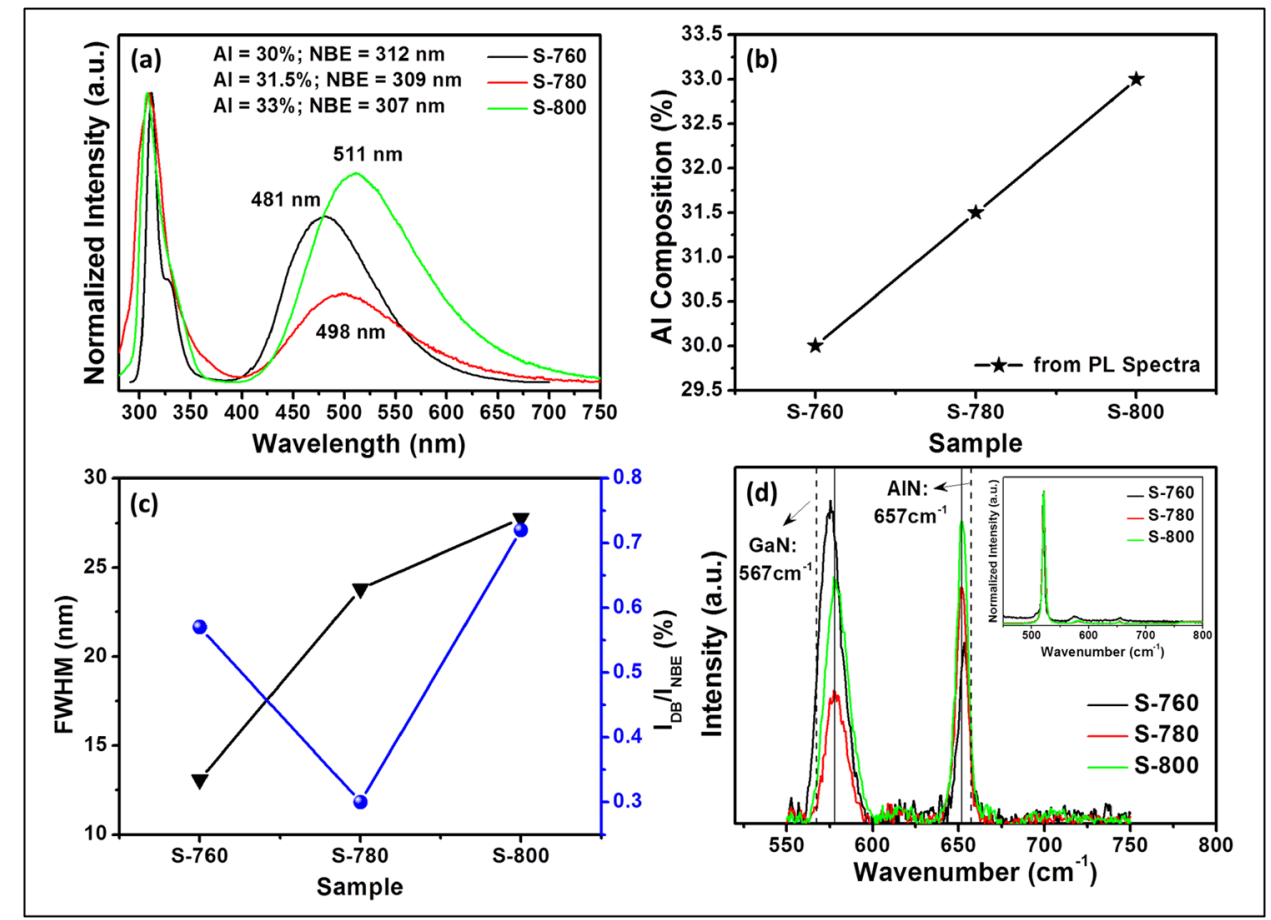


bandgap of the grown ternary AlGaN alloys. The NBE peaks are centered at 312,309 and $307 \mathrm{~nm}$ with error of $\pm 0.5 \mathrm{~nm}$ for samples S-760, S-780 and S-800, respectively.

Further, a shoulder peak to NBE emission was prominently observed at around $327 \mathrm{~nm}$ in S-760 (Fig. a) which is ascribed to transitions from shallow donor to shallow acceptor states known as Donor Accepter Pair (DAP) transitions in the grown AlGaN films. This characteristic can be attributed to an exciton bound to a native defect related shallow acceptor states such as any vacancy-related complex [31]. Though, a more prominent DAP could also exist in NBE peak's envelope of other AIGaN films in S-780 and S-800 which resulted in broadening of the NBE emission band. This enhanced DAP emission could be attributed to either increased III-group vacancies or influenced by the unintentional doping from the substrate during the growth into the nitride layer $[32,33]$. Besides, a broad emission band centered at around 481, 498 and $511 \mathrm{~nm}$ in samples S-760, S-780 and S-800, respectively is clearly noticed which is related to the defect states within the energy bandgap of the grown AIGaN films [34]. It can be clearly observed that the defect band is significantly suppressed for the film grown in S-780 sample. Next, the bandgap obtained from the NBE emission has been used for evaluating the Al composition ( $\mathrm{x}$ ) by using Vegard's law, [35]

$E_{g}^{A / G a N}=x \cdot E_{g}^{A I N}+(1-x) E_{g}^{G a N}+b . x \cdot(1-x)$

where $x$ is the $\mathrm{Al}$ mole fraction and $\mathrm{b}$ is the bowing parameter, $b=1.3$ in alloy $\mathrm{Al}_{x} \mathrm{Ga}_{1-\mathrm{x}} \mathrm{N}$ [36]. The evaluated composition values are plotted against the sample in Fig. $6 \mathrm{~b}$ and exhibited an incrementing trend with increasing buffer growth temperature. This suggests that buffer temperature and crystalline quality may lead to slight changes in the bandgap of the material. The Al composition is $30,31.5$ and $33 \%$ for S-760, S-780 and S-800, respectively. It was reported that the growth rate significantly affects the $\mathrm{Al}$ incorporation efficiency in AIGaN growth $[37,38]$. Thus, the increased Al incorporation from S-760 to S-800 samples could be related to different growth rates of AIGaN with varied AIN buffer growth quality. Although, these values are found to be slightly different from the HRXRD results that could be due to strain present in the grown films which have lead towards this deviation in the HRXRD results. Furthermore, it is known that the FWHM of NBE emission band (Fig. $6 \mathrm{C}$ ) determines the quality of grown epitaxial film, thus, the changes in buffer growth temperatures resulted in varying the quality of grown AIGaN films with least FWHM for sample S-760, which is found to be increased with increasing buffer growth temperature. This could be correlated to the enhanced DAP band existing along with NBE in complete peak envelope of S-780 and S-800.

On the other hand, Fig. $6 \mathrm{c}$ shows the change in ratio of defect band and NBE band extracted from the obtained experimental PL results of each sample. It was observed that the $I_{D B} / I_{N B E}$ value is least for the sample $S-780$ which signifies high quality AIGaN growth with least amount of trap/defect states in the energy bandgap. Therefore, although the FWHM is slightly higher for AIGaN film grown in S-780 still, the intensity ratio of defect band to NBE is prominently reduced compared to other two growths carried out in S-760 and S-800. Conclusively, the PL analysis recommend that the optical properties of AIGaN film grown in S-780 is superlative compared to the other grown samples and thus, it is highly expected to yield better device efficiency. The optical properties are further analyzed by performing micro-Raman spectroscopy. The Raman measurements have been used to evaluate the energies of phonon modes present in $\mathrm{Al}_{x} \mathrm{Ga}_{1-\mathrm{x}} \mathrm{N}$ ternary alloy. Inset of Fig. $6 \mathrm{~d}$ shows the micro-raman spectra obtained for a whole range of $450-800 \mathrm{~cm}^{-1}$ which yielded a highly dominant Si substrate peak at around $521.5 \mathrm{~cm}^{-1}$ in all the AIGaN/AIN/Si heterostructures. A closer examination unveils the existence of two modes each one of GaN-like and AIN-like mode branches from the PAMBE grown samples S-760, S-780 and S-800. This implicates two-mode behavior for $\mathrm{E}_{2}$ (high) mode of AIGaN [39]. For deeper investigation, the experimental data acquired from the Raman spectra is enlarged from $550-750 \mathrm{~cm}^{-1}$ as shown in Fig. 6 d. It was perceived that apart from sample S-760, the AIGaN grown in other two samples i.e. S-780 and S-800 almost coincides at same GaN-like and AIN-like $E_{2}$ (high) mode which suggests nearly same Al composition in AIGaN grown in samples S-780 and S-800 compared to S-760. The two mode behavior could be responsible for variation in the composition evaluation from HRXRD and the Raman phonon frequencies.

In summary, AlGaN film with high crystalline epitaxial quality has been grown by optimizing different AIN buffer layer growth conditions on $\mathrm{Si}$ (111) substrate using PAMBE. The AIN buffer has been grown under varied growth temperatures $\left(760^{\circ} \mathrm{C}\right.$ to $\left.800^{\circ} \mathrm{C}\right)$ wherein, a highly single crystalline AIGaN film with comparatively lowest XRC FWHM value of 2196 arcsec was witnessed when AIN buffer was grown at $780^{\circ} \mathrm{C}$. The quality of AIN buffer layer is found to impact the growth properties as well as the Al incorporation in the grown AlGaN films. The morphological analysis reveals highly smooth surface morphology with least surface defect states. Then, the optical properties were analyzed to gauge the influence of buffer layer on the AIGaN films where, the RT-PL analysis of sample S-780 revealed a bandgap value of $309 \mathrm{~nm}$ resulting in $31.5 \% \mathrm{Al}$ composition evaluated using the Vegard's law. Along with this, 
the defect band to NBE band ratio $\left(\mathrm{I}_{\mathrm{DB}} / \mathrm{I}_{\mathrm{NBE}}\right)$ is significantly reduced from 0.57 to 0.3 when buffer growth temperature increased from 760 to $780^{\circ} \mathrm{C}$. While, the $\mathrm{I}_{\mathrm{DB}} / \mathrm{I}_{\mathrm{NBE}}$ ratio again increased to 0.72 when the buffer temperature increases to $800{ }^{\circ} \mathrm{C}$. Therefore, the structural, morphological and optical properties divulges that the AIGaN film grown in sample S-780 (where buffer growth temperature is $780^{\circ} \mathrm{C}$ ) is highly crystalline in nature with minimum defects states. Conclusively, this study determines that the buffer layer temperature plays significant role in controlling the structural and optical properties of AIGaN layer grown over it.

Acknowledgement We gratefully acknowledge Director, CSIR-NPL, New Delhi, for his encouragement and support. Neha would like to acknowledge CSIR for providing financial assistance via CSIRResearch Associateship.

\section{Compliance with ethical standards}

Conflict of interest The authors declare no financial interest.

Open Access This article is licensed under a Creative Commons Attribution 4.0 International License, which permits use, sharing, adaptation, distribution and reproduction in any medium or format, as long as you give appropriate credit to the original author(s) and the source, provide a link to the Creative Commons licence, and indicate if changes were made. The images or other third party material in this article are included in the article's Creative Commons licence, unless indicated otherwise in a credit line to the material. If material is not included in the article's Creative Commons licence and your intended use is not permitted by statutory regulation or exceeds the permitted use, you will need to obtain permission directly from the copyright holder. To view a copy of this licence, visit http://creativecommons .org/licenses/by/4.0/.

\section{References}

1. Upadhyay KT, Chattopadhyay MK (2021) Sensor applications based on AlGaN/GaN heterostructures. Mater Sci Eng, B 263:114849

2. Dimkou I, Harikumar A, Ajay A, Donatini F, Bellet-Amalric E, Grenier A, den Hertog MI, Purcell ST, Monroy E (2020) Design of AIGaN/AIN Dot-in-a-Wire Heterostructures for Electron-Pumped UV Emitters. Physica status solidi (a) 217(7):1900714

3. Lingaparthi R, Dharmarasu N, Radhakrishnan K, Agrawal M (2020) In-situ stress evolution and its correlation with structural characteristics of GaN buffer grown on Si substrate using AIGaN/ AIN/GaN stress mitigation layers for high electron mobility transistor applications. Thin Solid Films 708:138128

4. Alaie Z, Mohammad Nejad S, Yousefi MH (2015) Recent advances in ultraviolet photodetectors. Mater Sci Semicond Process 29:16-55

5. Liu L, Edgar JH (2002) Substrates for gallium nitride epitaxy. Mater Sci Eng: R: Reports 37:61-127

6. Vittoz S, Rufer L, Rehder G, Heinle U, Benkart P (2010) Analytical and numerical modeling of AIGaN/GaN/AIN heterostructure based cantilevers for mechanical sensing in harsh environments. Procedia Eng 5:91-94

7. Carey P, Ren F, Bae J, Kim J, Pearton S (2020) Wide Bandgap Semiconductor-Based Electronics. IOP Publishing, Bristol
8. Aldalbahi A, Li E, Rivera M, Velazquez R, Altalhi T, Peng X, Feng PX (2016) A new approach for fabrications of SiC based photodetectors. Scientific Reports 6:23457

9. Jmerik V, Nechaev D, Rouvimov S, Ratnikov V, Kop'ev P, Rzheutski M, Lutsenko E, Yablonskii G, Aljohenii M, Aljerwii A, Alyamani A, Ivanov S (2015) Structural and optical properties of PA MBE AIGaN quantum well heterostructures grown on c-Al2O3 by using flux- and temperature-modulated techniques. J Mater Res 30:2871-2880

10. Koblmuller G, Chu RM, Raman A, Mishra UK, Speck JS (2010) High-temperature molecular beam epitaxial growth of AIGaN/ GaN on GaN templates with reduced interface impurity levels. J Appl Phys 107:043527

11. Shim KH, Myoung JM, Gluschenkov OV, Kim C, Kim K, Yoo MC, Kim S, Turnbull DA, Bishop SG (2011) Growth and Characterization of AlGaN/GaN Heterostructures with Multiple Quantum Wells by PAMBE. MRS Proceedings 423:347

12. Ganguly S, Verma J, Xing H, Jena D (2014) Plasma MBE growth conditions of AlGaN/GaN high-electron-mobility transistors on silicon and their device characteristics with epitaxially regrown ohmic contacts. Appl Phys Express 7:105501

13. Pramanik P, Sen S, Singha C, Roy AS, Das A, Sen S, Bhattacharyya $A$ (2016) Compositional inhomogeneities in AIGaN thin films grown by molecular beam epitaxy: Effect on MSM UV photodetectors. J Appl Phys 120:144502

14. Pau JL, Monroy E, Munoz E, Naranjo FB, Calle F, Sanchez-Garcia MA, Calleja E (2001) AIGaN photodetectors grown on Si(111) by molecular beam epitaxy. J Cryst Growth 230:544-548

15. Ristic J, Sanchez-Garcia MA, Calleja E, Sanchez-Paramo J, Calleja JM, Jahn U, Ploog KH (2002) AIGaN Nanocolumns Grown by Molecular Beam Epitaxy: Optical and Structural Characterization. Physica status solidi (a) 192:60-66

16. Liaw HM, Venugopal R, Wan J, Melloch MR (2001) Influence of the AIN buffer layer growth on AIGaN/GaN films deposited on (111)Si substrates. Solid-State Electronics 45:417-421

17. Iliopoulos E, Moustakas TD (2002) Growth kinetics of AIGaN films by plasma-assisted molecular-beam epitaxy. Appl Phys Lett 81:295-297

18. Rudziński M, Zlotnik S, Wójcik M, Gaca J, Janicki Ł, Kudrawiec $\mathrm{R}$ (2021) Mask-free three-dimensional epitaxial growth of IIInitrides. J Mater Sci 56(1):558-569

19. Mazumder B, Kaun SW, Lu J, Keller S, Mishra UK, Speck JS (2013) Atom probe analysis of AIN interlayers in AIGaN/AIN/ GaN heterostructures. Appl Phys Lett 102:111603

20. Aggarwal N, Krishna S, Kumar Jain S, Mishra M, Maurya KK, Singh S, Kaur M, Gupta G (2019) Microstructural evolution of high quality AIN grown by PAMBE under different growth conditions. Mater Sci Eng: B 243:71-77

21. Pandey A, Dutta S, Prakash R, Raman R, Kapoor AK, Kaur D (2018) Growth and Comparison of Residual Stress of AIN Films on Silicon (100), (110) and (111) Substrates. J Electron Mater 47:1405-1413

22. Norris DA, Rodriguez MA, Fukuda SK, Snyder RL (2013) X-Ray Powder Data for alpha-Si3N4. Powder Diffr 5:225-226

23. Ozen S, Senay V, Pat S, Korkmaz S (2015) Investigation on the morphology and surface free energy of the AIGaN thin film. $J$ Alloy Compd 653:162-167

24. Aggarwal N, Krishna S, Goswami L, Gupta G (2021) Inclination of screw dislocations on the performance of homoepitaxial GaN based UV photodetectors. Mater Sci Eng, B 263:114879

25. Z. Yang, C. Bingzhen, P. Na, Z. Lu, Y. Cuibai, P. Xu, Y. Shun, W. Zhiyong, Growth of AlGaN Film on Si (111) Substrate, in: MATEC Web of Conferences, Shanghai, China, 2016.

26. Moram MA, Vickers ME (2009) X-ray diffraction of III-nitrides. Rep Prog Phys 72:036502 
27. Aggarwal N, Krishna S, Goswami L, Mishra M, Gupta G, Maurya KK, Singh S, Dilawar N, Kaur M (2015) Extenuation of Stress and Defects in GaN Films Grown on a Metal-Organic Chemical Vapor Deposition-GaN/c-Sapphire Substrate by PlasmaAssisted Molecular Beam Epitaxy. Cryst Growth Des 15:2144

28. Tetzner H, Sana P, Klesse WM, Capellini G, Schubert MA, Thapa SB, Storck P, Schroeder T, Zoellner MH (2020) Carbon related hillock formation and its impact on the optoelectronic properties of GaN/AIGaN heterostructures grown on $\mathrm{Si}(111)$. Appl Phys Lett 116:252101

29. Singh VK, Pandey A, Tyagi R (2020) Optimization of AIN spacer layer in MOVPE grown AIGaN/AIN/InGaN/GaN high electron mobility heterostructure. AIP Conf Proc 2220:090026

30. Rathkanthiwar S, Kalra A, Remesh N, Bardhan A, Muralidharan $R$, Nath DN, Raghavan S (2020) Impact of pits formed in the AIN nucleation layer on buffer leakage in GaN/AIGaN high electron mobility transistor structures on Si (111). J Appl Phys 127:215705

31. Reshchikov MA, Morkoç $\mathrm{H}$ (2005) Luminescence properties of defects in GaN. J Appl Phys 97:061301

32. Paskova T, Arnaudov B, Paskov PP, Goldys EM, Hautakangas S, Saarinen K, Södervall U, Monemar B (2005) Donor-acceptor pair emission enhancement in mass-transport-grown GaN. J Appl Phys 98:033508

33. Le LC, Zhao DG, Jiang DS, Wu LL, Li L, Chen P, Liu ZS, Zhu JJ, Wang $\mathrm{H}$, Zhang SM, Yang H (2012) Effect of light Si-doping on the near-band-edge emissions in high quality GaN. J Appl Phys 112:053104
34. Krishna S, Aggarwal N, Gundimeda A, Sharma A, Husale S, Maurya KK, Gupta G (2019) Correlation of donor-acceptor pair emission on the performance of GaN-based UV photodetector. Mater Sci Semicond Process 98:59-64

35. Vurgaftman I, Meyer JR (2003) Band parameters for nitrogencontaining semiconductors. J Appl Phys 94:3675-3696

36. Angerer $H$, Brunner $D$, Freudenberg $F$, Ambacher $O$, Stutzmann M, Hopler R, Metzger T, Born E, Dollinger G, Bergmaier A, Karsch $\mathrm{S}$, Korner HJ (1997) Determination of the Al mole fraction and the band gap bowing of epitaxial AlxGa1b xN films. Appl Phys Lett 71:1504-1506

37. Deng Y, Zhao DG, Le LC, Jiang DS, Wu LL, Zhu JJ, Wang H, Liu ZS, Zhang SM, Yang H, Liang JW (2011) Relationship between the growth rate and $\mathrm{Al}$ incorporation of $\mathrm{AIGaN}$ by metalorganic chemical vapor deposition. J Alloy Compd 509:748-750

38. Li D-B, Aoki M, Katsuno T, Miyake H, Hiramatsu K, Shibata $T$ (2007) Influence of growth conditions on Al incorporation to AlxGa1b xN ( $x>04)$ grown by MOVPE. J Cryst Growth 298:372-374

39. Harima H (2002) Properties of $\mathrm{GaN}$ and related compounds studied by means of Raman scattering. J Phys: Condens Matter 14:R967-R993

Publisher's Note Springer Nature remains neutral with regard to jurisdictional claims in published maps and institutional affiliations. 